\title{
The Rhizoctonia solani RsLysM and RsRIpA effector proteins contribute to virulence by suppressing chitin-triggered immunity and hypersensitive response
}

Fredrik Dölfors ${ }^{1}$, Louise Holmquist ${ }^{1,2}$, Panagiotis N. Moschou ${ }^{1}$, Christina Dixelius ${ }^{1}$ and Georgios Tzelepis* ${ }^{1}$

1 Swedish University of Agricultural Sciences, Department of Plant Biology, Uppsala BioCenter, Linnean Center for Plant Biology, P.O. Box 7080, S75007 Uppsala, Sweden

${ }^{2}$ MariboHilleshög Research AB, Säbyholmsvägen 24, S-26191, Landskrona, Sweden

*For correspondence: Georgios.Tzelepis@slu.se,

\begin{abstract}
Rhizoctonia (in Greek "root-killer") species and particularly $R$. solani attacks a broad range of plant species and crops. It belongs to Basidiomycota and is a soil-borne pathogen causing mainly damping-off diseases of seedlings and root rot, although it can infect plants in any stage. Despite the severity of this disease, many aspects in $R$. solani infection biology still remain to be elucidated. Here we investigated the role of two effector candidates, predicted from the genome of a $R$. solani AG2-2IIIB strain that uses sugar beet as a host. Gene expression analysis showed that genes encoding for a LysM effector and a rare lipoprotein-A-like protein (RsRlpA) were induced upon early infection stages. When heterologous expressed in Cercospora beticola the two genes contributed to virulence. The RsLysM effector showed chitinbinding affinity and suppression of chitin-triggered immunity but could not protect hyphae from hydrolysis. The RsRlpA effector suppressed hypersensitive response in Nicotiana benthamiana leaves. Overall, this study provides us with valuable information on $R$. solani infection biology, implying that this organism relies on mechanisms similar to hemibiotrophic pathogens in order to establish a successful infection.
\end{abstract}

Keywords: Cercospora beticola, effectors, heterologous expression, LysM, RlpA protein, sugar beet 


\section{Introduction}

Rhizoctonia solani Kühn is an important soil-borne pathogen causing substantial yield losses in a wide range of crops including cereals, soybean, potato and sugar beet (Adams, 1988). It is responsible for damping-off disease in many hosts (Anderson et al., 1982). It incites crown and root rot in sugar beet, leaf spot and root rot in tobacco, sheath blight in rice and black scurf in potato (Ceresini et al., 2002; Bernardes-de-Assis et al., 2009; Buhre et al., 2009; Gonzalez et al., 2011). The fungus produces persistent sclerotia, which are the major infestation source together with fungal-infested plant debris in the soil. Rhizoctonia solani commonly infects roots and hypocotyls but all types of plant organs can be colonized by the mycelia. The sexual stage (Thanatephorus cuccumeris) is extremely rare (Adams and Butler, 1983) and conidia formation is lacking. This pathogen is classified into different anastomosis groups (AGs) based on hyphal cell wall fusion between different isolates. Some AGs are further divided into subgroups based on host range, colony morphology, pathogenicity, zymogram patterns and other characteristics (Ogoshi et al., 1987).

Despite the economic impact of $R$. solani, little is known about mechanisms behind fungal colonization and growth on host tissue. Hypothesis on gene function is difficult to test since $R$. solani is not amenable to molecular manipulations. A general view is that $R$. solani hyphae adhere to the host plant surface and enter the outer cell layers by the formation of penetration cushions (Gonzalez et al., 2011), a process followed by the growth of invasive hyphae that ramify through the host tissue.

Secreted proteins promoting disease were first observed in human pathogenic bacteria (Preston, 2007). As more genomes of plant pathogens have been generated the prediction of secreted proteins or otherwise transported molecules have increased substantially. Evolution of effectors is a strategy by pathogens to manipulate plant defenses to establish a successful infection (Vleeshouwers and Oliver, 2014; Lo Presti et al., 2015). A plethora of strategies are employed; effectors suppress and block early plant defense responses such as hypersensitive response (HR) (Hemetsberger et al., 2012), stealth hyphae from recognition or protect them from plant chitinases (de 
Jonge and Thomma, 2009, van den Burg et al., 2006) or promote necrosis (Qutob et al., 2006). Key molecules acting in various pathways can also be hijacked impacting gene activation and hormones (Weiberg et al., 2013; Ma and $\mathrm{Ma}, 2016)$. Many aspects on effector function during plant-microbe interactions remain unclear and host targets are most likely more than one. Genomic data are presently available from five different $R$. solani strains with cereal and dicot plant hosts (Cubeta et al., 2014; Hane et al., 2014; Wibberg et al., 2013, 2016a,b; Zheng et al., 2013). In an AG8 strain, attacking both monocots and dicots, a xylanase and a protease inhibitor 19 induced cell death when expressed in Nicotiana benthamiana (Anderson et al., 2017). Similarly, cell death induction was also observed on rice, maize and soybean leaves, when purified candidate effectors from an AG1-1A strain, causing rice sheath blight, were evaluated (Zheng et al., 2013).

Sugar beet is mainly attacked by the AG2-2IIIB strain. The disease can appear in different forms and with different symptoms; root and crown rot, damping off or as foliar blight (Bolton et al., 2010). The demand for high yielding sugar beet cultivars with resistance to $R$. solani is increasing. Effects of a milder and more humid climate are seen in temperate regions influencing the length of crop seasons and simultaneously allow pathogens to multiply for longer periods. Moreover, the extent of $R$. solani AG2-2IIIB soil inoculum is expected to increase in regions particularly where sugar beet and maize are overlapping in the crop rotation schemes since maize can act as a host and thus propagate the pathogen (Buddemeyer et al., 2004; Schulze et al., 2016). The $R$. solani AG2-2IIIB strain has a predicted genome size of $56.02 \mathrm{Mb}$ and 11,897 protein-encoding genes (Wibberg et al., 2016a,b). Here we refined the dataset and identified 11 AG2-2IIIB-specific candidates. A rare lipoprotein-Alike (RsRlpA) protein and the chitin-binding lysin motif (RsLysM) effector were highly induced in infected sugar beet seedlings. In planta assays using Cercospora beticola as a heterologous expression system harboring the two $R$. solani genes showed increased virulence. The RsLysM effector was able to suppress chitin-triggered immunity, while the RsRlpA effector was able to suppress the hypersensitivity response (HR) upon transient expression on $N$. benthamiana leaves. These data give us valuable information regarding the 
different mechanisms that $R$. solani deploys upon the infection process and it seems that this pathogen does not only rely on necrosis-induced effectors.

\section{Materials and Methods}

\section{Fungal isolates and sequence analysis}

Rhizoctonia solani AG2-2IIIB isolate BBA 69670 (DSM 101808) (Wibberg et al., 2016a) was used in this study. $R$. solani inoculum for soil infestation was prepared on media containing perlite, corn flour, and water. Cercospora beticola isolate Ty1 was grown on potato dextrose agar plates at $22^{\circ} \mathrm{C}$ in darkness and sporulation was induced on tomato growth extract medium. Small cysteine-rich and secreted proteins were retrieved from $R$. solani AG22IIIB genome (Wibberg et al., 2016b). Presence of conserved domains was searched for by using the SMART 6.0 tool (Letunic et al., 2009), followed by SignalP 4.1 for signal peptide prediction (Petersen et al., 2011). The Phyre2 server was used for protein structure prediction (Kelley et al., 2015).

\section{Quantitative reverse transcriptase - PCR (qRT-PCR)}

Three-week old sugar beet plants were transplanted in soil infested with $R$. solani mycelia (10:1 ratio of fresh soil: inoculum). Total RNA was extracted from the plants at 4,5,6 and 7 days post inoculation (dpi) using the RNeasy Plant Mini Kit (Qiagen) according to manufacturer's instructions, while $R$. solani mycelia grown in potato dextrose broth were used as fungal control. qRT-PCR was run and primers are listed in Table S1. Expression was normalized by $R$. solani G3PDH expression (Chamoun et al., 2015) and relative expression values were calculated according to the $2^{-\Delta \Delta C t}$ method (Livak and Schmittgen, 2001).

\section{Cercospora beticola overexpression strains and virulence assays}

Overexpression vectors were designed using the In-Fusion HD cloning kit (Takara Bio). The RsLysM and RsRlpA sequences were PCR amplified from $R$. solani cDNA using high fidelity Phusion Taq polymerase (Thermo Scientific). The destination vector was the pRFHUE-eGFP, conferring resistance to hygromycin (Crespo-Sempere et al., 2011). The PgdpA: effector construct was tagged with promoter with GFP in the C-terminus and 
transformed to $C$. beticola using an Agrobacterium-mediated protocol (Utermark and Karlovsky, 2008). Primers are listed in Table S1. Three independent colonies expressing the correct effector genes were selected for further analysis.

For virulence assays, leaves of 3-week-old sugar beet plants were inoculated with $10^{5} \mathrm{C}$. beticola conidia $/ \mathrm{ml} \mathrm{H}_{2} \mathrm{O}$ from overexpressed, empty vector and wild-type (Ty1) strains. Lesion area was monitored at $7 \mathrm{dpi}$, and quantified using the ImageJ software version 1.51n (National Institute of Health, USA). Total genomic DNA was extracted from the diseased plant material using a CTAB-mediated protocol (Möller et al., 1992). Fungal DNA was quantified using the $C$. beticola actin (act) reference gene and normalized with $B$. vulgaris elongation factor (elf-1) gene. Primers are listed in Table S1.

\section{Confocal microscopy and hypersensitive response assay}

The RsRlpA effector sequence was cloned into pENTR/D-TOPO Gateway vector (Thermo Fisher Scientific), and entered to pGWB605 or pGWB660 binary vectors, tagged with C-terminus GFP or RFP fluorescence proteins respectively, driven by the $35 \mathrm{~S}$ promoter and transformed into Agrobacterium tumefaciencs C58C1. Overnight cultures were used for Agro-infiltration on 4week old $N$. benthamiana leaves. Protein cellular localization was monitored by using a Zeiss LSM 800 confocal microscope. For the hypersensitive response assay, the $R s R I p A$ gene was entered to the pGWB602 binary vector without tag, driven by the $35 \mathrm{~S}$ promoter, followed by Agro-infiltration in transgenic $N$. benthamiana, expressing the Cf-4 receptor protein from tomato plants (Joosten et al., 1997). A HR resonce was triggered 24 hrs after Agroinfiltration with the Cladosporium fulvum Avr4 effector protein in O.D 0.03 (Joosten et al., 1994). The HR was evaluated using a scale from 0-3, ranging from no symptoms (0) to severe symptoms (3).

\section{Expression of RsLysM in Pichia pastoris}

The RsLysM protein was cloned in the pPic9 expression vector with $\mathrm{N}$ terminal His tag and transformed into the $P$. pastoris strain GS113. Primers are listed in Table S1A positive clone was cultured in a Bioflo 300 fermenter (Rooney et al., 2005). His-tagged protein was purified using a Ni-NTA column 
(Qiagen) and final protein concentration was determined spectrophotometrically at $280 \mathrm{~nm}$, and concentration analyzed by a Pierce BCA Protein assay kit (Thermo Fisher Scientific).

\section{Affinity precipitation, reactive oxygen species and hyphal protection assay}

A chitin-binding assay of the RsLysM effector protein was performed. Pure protein was incubated with insoluble polysaccharides (crab shell chitin, chitosan, xylan or cellulose) and analyzed on SDS polyacrylamide gel (van de Burg et al., 2006). For suppression of chitin-induced oxidative burst of reactive oxygen species (ROS) assay, the protocol by de Jonge et al. (2010) was used. Here leaf discs were inoculated with assay buffer with/without chitin oligomers (GIcNAc) ${ }_{6}$ and the suppression of ROS was tested using 10-20 $\mu \mathrm{M}$ RsLysM protein. For hyphal protection assay a protocol described previously by Kohler et al., (2016) was used. Briefly, the protein was incubated with germinated conidia from Trichoderma virens. Then bacterial chitinase (Sigma) and $10 \mathrm{U}$ zymolyase (Zymo Research) were added. The Avr4 effector protein $(5 \mu \mathrm{M})$ and BSA were used as positive and negative controls respectively. Images were taken 6 hrs post inoculation (hpi).

\section{Results}

\section{RsLysM and RsRIpA are two effector candidates induced upon early- stage infection}

In the previous genome analysis of the $R$. solani AG2-2IIIB isolate, 126 predicted secreted and cysteine-rich proteins were identified (Wibberg et al., $2016 b)$. To further reduce the list of effector-candidates only small proteins (<400 amino acids) were kept resulting in 61 predicted sequences. Eleven candidates appeared to be unique in this isolate. Analysis of sugar beet plants grown in $R$. solani $(R s)$ infested soil revealed elevated transcript levels of RsLysM (RSOLAG22IIIB_4067) and RsRIpA (RSLAG22IIIB_8473) genes already at 4 dpi suggesting an important role during $R$. solani plant host colonization (Figure 1). RsRlpA was predicted to encode a rare lipoprotein $\mathrm{A}$ $(\mathrm{R} \mid \mathrm{pA})$-like domain protein, previously described in bacteria (Gerding et al., 2009). Fungal LysM effectors are versatile proteins that occur in a plethora of 
species with divergent lifestyles. The general function of LysM molecules are to evade plant recognition but may also have other roles, for instance it is required for the appresorium function in Colletotrichum higginsianum (Takahara et al., 2016).

\section{RsLysM and RsRIpA promote virulence upon heterologous expression in Cercospora beticola}

The amino acid sequences of RsLysM and RsRlpA were analyzed for conserved domains. The SMART tool predicted two CBM50 modules (IPR002482; LysM peptidoglycan binding) in the RsLysM effector and a conserved double-psi beta-barrel (IPR036908; DPBB fold) in RsRlpA (Figure 2A). Structural prediction of RsRlpA using the Phyle2 resulted in: 39\% homology to a bacterial cellulose binding protein, $34 \%$ to a papain-like inhibitor, $27 \%$ to expansin, $26 \%$ to kiwellin protein and $26 \%$ to barwin-like endoglucanase. Both RsLysM and RsRIpA proteins contain an ER signal peptide at the N-terminus, according to SingalP.

To test if the two effector-candidates are involved in virulence, we heterologously expressed them in the sugar beet hemibiotroph fungal pathogen $C$. beticola. Using qRT-PCR we confirmed the expression of these genes in $R s R l p A+$ and $R s L y s M+$ strains (Figure S1). Phenotypic analysis on these strains displayed no difference in morphology, growth rate or conidiation in comparison to wild-type (Ty1). The $R s L y s M+C$. beticola strains, displayed significantly larger necrotic lesions on sugar beet leaves, compared to wildtype and strains where only the empty vector was inserted (Figure 2B, C). In contrast, no clear phenotypic difference was observed in $R s R l p A+$ strains. Similarly, transient expression of RsRlpA in $N$. benthamiana did not induce cell death. The fungal biomass was significantly increased in comparison with wild-type and empty vector for both $R s L y s M+$ and $R s R l p A+$ strains, further supporting the role of these candidate effectors in host colonization (Figure 2D). The localization of RsLysM and RsRlpA was checked in C. beticola fungal hyphae. Both candidate effectors were localized to the cell periphery, and RsLysM was particularly observed in hyphal tips (Figure 3). 


\section{The RsRIpA effector suppresses hypersensitive response and is mainly localized to the plant plasma membrane}

Many plant pathogens rely on an initial short biotrophic stage to establish a successful infection and therefore secrete effectors to work as suppressors of HR (Stergiopoulos and de Wit, 2009). A hemibiotrophic stage has also been proposed for R. solani (Gonzalez et al., 2011). Thus, the ability of RsRlpA effector to suppress HR was investigated by exploiting the Avr4-Cf4 interaction that leads to severe HR symptoms in $N$. benthamiana. Our data suggest that in leaf areas where RsRIpA had been previously Agro-infiltrated, reduced HR was observed as compared to the area where only Avr4 was expressed (Figure 4). This observation suggests that RsRlpA potentially suppresses HR.

Despite GFP tagging in $R s R l p A+$ strains, we repeatedly failed to clearly observe the RsRlpA localization during the infection process in sugar beet leaves. Thus, to determine where this effector is localized, we transiently expressed it in $N$. benthamiana leaves, tagged with GFP or RFP at Cterminus. We observed that RsRlpA was mainly localized to the plasma membrane (Figure 5). Localization of RsRlpA was also observed in the nucleoplasm and ER. Plasmolysis of the $N$. benthamiana leaves, using mannitol, supported the plasma membrane localization.

\section{The RsLysM effector binds to chitin}

Previous analyses of LysM effector proteins from plant pathogenic ascomycetes have demonstrated their ability to bind chitin (de Jonge et al., 2010; Marshall et al., 2011; Kombrink et al., 2017). Whether LysM effectors from basidiomycetes have a similar chitin-binding affinity is unknown. We expressed RsLysM in yeast and purified the protein, which was used for the chitin-binding assay. The $R$. solani LysM protein was able to interact with all tested forms of chitin, such as chitin beads, crab shell chitin and chitosan (Figure 6A). No precipitation with other polysaccharides such as xylan and cellulose was detected. Together the data suggests that RsLysM is an active chitin-binding effector protein, similar to other already characterized LysM effectors from filamentous ascomycetes. 


\section{The RsLysM effector suppresses chitin-triggered immunity but does not protect hyphae from degradation}

It is known that LysM effectors stealth filamentous ascomycetes to avoid plant immunity responses triggered by chitin (de Jonge et al., 2010; Marshall et al., 2011; Mentlak et al., 2012; Kombrink et al., 2017). To investigate whether the RsLysM effector also prevent plant chitin-triggered immunity, $N$. benthamiana leaves were treated with chitin oligomers $(\mathrm{GlcNAc})_{6}$, which led to reactive oxygen species (ROS) burst (Figure 6B), suggesting that RsLysM displays similar non-recognition function as seen in other pathosystems. Certain LysM effectors are able to protect fungal hyphae from chitinolytic activity (Marshall et al., 2011; Kombrink et al., 2017) similar to the Avr4 effector from C. fulvum (van den Burg et al., 2006). To investigate whether the RsLysM displays similar function, germinated conidia from $T$. viride were mixed with pure RsLysM protein, while Avr4 and BSA were used as positive and negative controls respectively. RsLysM was unable to protect fungal hyphae against degradation from bacterial chitinases and zymolyases, in contrast to Avr4 (Figure 6C).

\section{Discussion}

Rhizoctonia solani is a pathogen commonly described as a saprophyte that is able to switch to pathogenic endotrophic growth thriving on dead or dying plant debris/cells. A complete understanding on the switch of lifestyles from endophytic to a parasitic form is still unclear. Theories underlying the phenomenon are about; an imbalance in nutrient exchange between plant and microorganism, single gene mutations as shown in Colletotrichum magna, and light-induced production of $\mathrm{H}_{2} \mathrm{O}_{2}$ (Redman et al., 2001; Alvarez-Loayza et al., 2011; Kuo et al., 2014). Loss of cell wall-degrading enzymes and high activity of certain genes lacking functional annotation has been suggested to at least partly explain the switch to the symbiotic lifestyle of ectomycorrhiza (Kohler et al., 2015). To plan for targeted pathogen control measures mechanisms behind these transitions are essential to be clarified.

The LysM domain is ubiquitous and can be found across all kingdoms. The LysM module recognizes the GlcNAc-X-GlcNAc sequence in polysaccharides (Buist et al., 2008) and details on the binding characteristics are clarified 
(Mesnage et al., 2014). In prokaryotes, LysM motifs bind to peptidoglycan in bacterial cell walls, whereas LysM in eukaryotes mainly bind to the abundant chitin polymer. In parallel have numerous LysM receptors been identified for example one that binds to nodulation factors important for nodule development in legumes and thereby promote nitrogen fixation (Broghammer et al., 2012). In plant - pathogen interactions, a wealth of knowledge on LysM derives from pathogenic fungal species, (de Jonge et al., 2010; Marshall et al., 2011; Mentlak et al., 2012; Kombrink et al., 2017).

In this study, we investigated the role of two candidate effectors predicted as singletons in the $R$. solani AG2-2IIIB strain (Wibberg et al., 2016b). The RsLysM effector gene showed induction upon early infection stage including suppression of ROS burst, which is in agreement with the previously published data from foliar pathogens such as C. fulvum and Mycosphaerella graminicola (de Jonge et al., 2010; Marshall et al., 2011). The situation looks different in Verticillium dahliae, where a lineage-specific LysM effector contributes to virulence on tomato but not on $N$. benthamiana or Arabidopsis (Kombrink et al., 2017).

Our $C$. beticola strains overexpressing the RsLysM displayed increased fungal biomass and necrotic lesions in infected sugar beet plants, strongly supporting its role in host colonization. Similarly, the lineage-specific Vd2LysM and Ecp6 effectors are required for full virulence of $V$. dahliae and $C$. fulvum respectively, since deletion of these genes led to reduced symptom development (Bolton et al., 2008; de Jonge et al., 2013). In contrast, deletion of three core LysM effectors from the $V$. dahliae JR2 strain, showed no changes in pathogen virulence (Kombrink et al., 2017), indicating functional differentiation among them. RsLysM did not protect fungal hyphae from degradation, which indicates that it is not involved in protecting the fungal cell wall from external hydrolytic enzymes. This finding is in analogy of the Epc6 LysM effector function (de Jonge et al., 2010). The situation looks different in some other plant-pathogen systems (Marshal et al., 2011; Kombrink et al., 2017), and suggests that hyphal protection is not a universal function for LysM effectors.

The RsRlpA candidate effector is a protein, containing a barwin-like double psi beta-barrel (DPBB) domain. This domain has a possible enzymatic 
function and it has been identified in various proteins, such as expansins, dehydrogenases, endo-glucanases and proteinases (Castillo et al., 1999). The function of the DPBB domain in filamentous fungi remains unknown, but proteins containing this domain were identified in the secretome analysis of virulent Pyrenophora teres f. teres isolates, causing net blotch disease in barley (Ismail and Able, 2016) and in Basidiomycota species causing brown and white rot (Pellegrin et al., 2015). The RsRlpA gene was induced at an early stage of sugar beet infection. Induction of a homolog to $R s R / p A$ in Magnaporthe oryzae is also active in invasive hyphae invaded the first plant cells, indicating a role during the biotrophic stage of this hemibiotrophic pathogen (Mosquera et al., 2009). The C. beticola RsRlpA overexpression strains, displayed increased fungal biomass in infected plants, and to our best of knowledge, this is the first report showing the contribution of this kind of protein in fungal virulence.

Although more studies are needed in order to characterize the RsRlpA enzymatic function and the HR suppression, this study gave us valuable information about the potential role of effectors in the AG2-2IIIB $R$. solani strain. It seems that this pathogen deploys effectors to manipulate important plant immunity mechanisms and it does not rely only to necrosis-induced effectors. We could also speculate that $R$. solani AG2-2IIIB needs an initial biotrophic stage crucial for a successful establishment of infection, implying a hemibiotrophic life style.

\section{Supplementary material}

Figure S1. Validation of $C$. beticola overexpression strains harboring the $R$. solani RsLySM and RsRIpA genes. Transcript from cDNA extracted from mycelia grown on PDA medium, for 7days. C. beticola wild type (WT) was used as negative control and act gene used as a positive control.

Tables S1. Primers used in the current study

\section{Acknowledgments}

The authors want to acknowledge: Prof. Bart Thomma, Peter Herfs, Nick Snelders and Hui Tian at Wageningen University, Phytopathology Lab, who 
contributed to RsLysM protein production, purification and provided us with $N$. benthamiana Cf-4 seeds, MariboHilleshög Research that provided C. beticola Ty1 strain and sugar beet seeds (breeding line 16045118 01), to Dr. Ioannis Stergiopoulos and Dr. Li Hung-Chen at UC Davis, Phytopathology Lab for helping us with the hyphal protection assay. This work was supported by grants from: the Swedish Research Councils VR and Formas, MariboHilleshög Research and the Swedish University of Agricultural Sciences.

\section{References}

Adams, G.C. (1988). Thanatephorus cucuemris (Rhizoctonia solani) a species complex of wide host range Advances in Plant Pathology 6, 535-592.

Adams, G.C. \& Butler, E.E. (1983). Influence of nutrition on the formation of basidia and basidiospores in Thanatephorus cucumeris. Phytopathology, 73, 147-151.

Alvarez-Loayza, P. et al. (2011). Light converts endosymbiotic fungus to pathogen, influencing seedling survival and niche-space filling of a common tropical tree, Iriartea deltoidea. PLoS One 6,e16386.

Anderson, J.P. et al. (2017). Comparative secretome analysis of Rhizoctonia solani isolates with different host ranges reveals unique secretomes and cell death inducing effectors. Scientific Reports 7, 10410.

Anderson, N.A. (1982). The Genetics and pathology of Rhizoctonia solani. Annual Review of Phytopathology 20, 329-347.

Bolton, M.D. et al. (2010). Temperature, moisture, and fungicide effects in managing Rhizoctonia root and crown rot of sugar beet. Phytopathology 100, 689-697.

Bolton, M.D. et al. (2008). The novel Cladosporium fulvum lysin motif effector Ecp6 is a virulence factor with orthologues in other fungal species. Molecular Microbiology 69, 119-36.

Bernardes-de-Assis, J. et al. (2009). Genetic structure of populations of the rice-infecting pathogen Rhizoctonia solani AG-1 IA from China. Phytopathology 99, 1090-1099.

Broghammer, A. et al. (2012). Legume receptors perceive the rhizobial lipochitin oligosaccharide signal molecules by direct binding. Proceedings National Academy of Sciences, USA 109, 13859-13864.

Buddemeyer, J. et al. (2004). Genetic variation in susceptibility of maize to Rhizoctonia solani (AG 2-2IIIB) - symptoms and damage under field conditions in Germany. Journal of Plant Diseases and Protection 111, 521-533.

Buhre, C. et al. (2009). Integrated control of root and crown rot in sugar beet: combined effects of cultivar, crop rotation, and soil tillage. Plant Disease 93, 155-161.

Buist, G. et al. (2008). LysM, a widely distributed protein motif for binding to (peptido)glycans. Molecular Microbiology 68, 838-847.

Castillo, R.M. et al. 1999. A six-stranded double-psi beta barrel is shared by several protein superfamilies. Structure with Folding \& Design 7, 227236. 
Ceresini, P.C. et al. (2002). Genetic structure of populations of Rhizoctonia solani AG-3 on potato in eastern North Carolina. Mycologia 94, 450460.

Chamoun, R. et al. (2015). Suppression subtractive hybridization and comparative expression of a pore-forming toxin and glycosyl hydrolase genes in Rhizoctonia solani during potato sprout infection. Molecular Genetics and Genomics 290, 877-900.

Crespo-Sempere, A. et al. (2011). Development of a green fluorescent tagged strain of Aspergillus carbonarius to monitor fungal colonization in grapes. International Journal of Food Microbiology 148, 135-40.

Cubeta, M.A. et al. (2014). Draft genome sequence of the plant-pathogenic soil fungus Rhizoctonia solani anastomosis group 3 strain Rhs1AP. Genome Announcments 2, e01072-14

de Jonge, $R$. et al. (2013). Extensive chromosomal reshuffling drives evolution of virulence in an asexual pathogen. Genome Reseaech 23, 1271-82.

de Jonge, R. \& Thomma, B.P.H.J. (2009). Fungal LysM effectors: extinguishers of host immunity? Trends in Microbiology 17, 151-157.

de Jonge, R. et al. (2010). Conserved fungal LysM effector Ecp6 prevents chitin-triggered immunity in plants. Science 329, 953-955.

Fradin, E.F. \& Thomma, B.P. (2006). Physiology and molecular aspects of Verticillium wilt diseases caused by $V$. dahliae and $V$. albo-atrum. Molecular Plant Pathology 7, 71-86.

Gerding, M.A., et al. (2009). Self-enhanced accumulation of FtsN at division sites and roles for other proteins with a SPOR Domain (DamX, DedD, and RlpA) in Escherichia coli cell constriction. Journal of Bacteriology 191, 7383-7401.

Gonzalez, M. et al. (2011). Tobacco leaf spot and root rot caused by Rhizoctonia solani Kuhn. Molecular Plant Pathology 12, 209-216.

Hane, J.K., et al. (2014). Genome sequencing and comparative genomics of the broad host-range pathogen Rhizoctonia solani AG8. PLoS Genetics 10, e1004281.

Hemetsberger, C. et al. 2012. The Ustilago maydis effector Pep1 suppresses plant immunity by inhibition of host peroxidase activity. Plos Pathogens 8, e1002684.

Ismail, I.A. \& Able, A.J. (2016). Secretome analysis of virulent Pyrenophora teres f. teres isolates. Proteomics 16, 2625-2636.

Joosten, M.H.A.J. et al (1994). Host resistance to a fungal tomato pathogen lost by a single base-pair change in an avirulence gene. Nature 367, 384-386.

Joosten, M.H.A.J. et al (1997). The biotrophic fungus Cladosporium fulvum circumvents Cf-4 mediated resistance by producing unstable AVR4 elicitors. Plant Cell 9, 367-379.

Kelley L.A. et al. (2015). The Phyre2 web portal for protein modeling, prediction and analysis. Nature Protocols 10, 845-858.

Kohler, A. et al. (2015). Convergent loss of decay mechanisms and rapid turnover of symbiosis genes in mycorrhizal mutualisms. Nature Genetics 47,410-415.

Kohler, A.C. et al. (2016). Structural analysis of an Avr4 effector ortholog offers insight into chitin binding and recognition by the Cf-4 receptor. Plant Cell 28, 1945-1965. 
Kombrink, A. et al (2017). Verticillium dahliae LysM effectors differentially contribute to virulence on plant hosts. Molecular Plant Pathology 18, 596-608.

Kuo, H.C. et al. (2014). Secret lifestyles of Neurospora crassa. Scientific Reports 4, 5135.

Letunic, I. et al. (2009). SMART 6: recent updates and new developments. Nucleic Acids Research 37, D229-32.

Livak, K.J. \& Schmittgen, T.D. (2001). Analysis of relative gene expression data using real-time quantitative PCR and the 2(-Delta Delta $\mathrm{C}(\mathrm{T})$ ) method. Methods 25, 402-408.

Lo Presti, L. et al. (2015). Fungal effectors and plant susceptibility. Annual Review of Plant Biology 66, 513-545.

Ma, K.W \& Ma, W. (2016). Phytohormone pathways as targets of pathogens to facilitate infection. Plant Molecular Biology 91, 713-725.

Marshall, R. et al. (2011). Analysis of two in planta expressed LysM effector homologs from the fungus Mycosphaerella graminicola reveals novel functional properties and varying contributions to virulence on wheat. Plant Physiology 156, 756-769.

Mentlak, T.A. et al. (2012). Effector-mediated suppression of chitin-triggered immunity by Magnaporthe oryzae is necessary for rice blast disease. Plant Cell 24, 322-335.

Mesnage, S. et al. 2014. Molecular basis for bacterial peptidoglucan recognition by LysM domains. Nature Communications 5,4269.

Möller, E.M. et al (1992). A simple and efficient protocol for isolation of high molecular weight DNA from filamentous fungi, fruit bodies and infected plant tissues. Nucleic Acids Research 20, 6115-6116.

Mosquera, G. et al (2009). Interaction transcriptome analysis identifies Magnaporthe oryzae BAS1-4 as biotrophy-associated Secreted proteins in rice blast disease. Plant Cell 21, 1273-1290.

Ogoshi, A. (1987). Ecology and pathogenicity of anastomosis and intraspecific groups of Rhizoctonia solani Kuhn. Annual Review of Phytopathology $25,125-143$.

Pellegri, N.C. et al (2015). Comparative analysis of secretomes from ectomycorrhizal fungi with an emphasis on small-secreted proteins. Frontiers in Microbiology 6, 1278.

Petersen, T.N. et al. (2011). SignalP 4.0: discriminating signal peptides from transmembrane regions. Nature Methods 8, 785-786.

Preston, G.M. (2007). Metropolitan microbes: Type III secretion in multihost symbionts. Cell Host \& Microbe 2, 291-294.

Qutob, D. et al. (2006). Phytotoxicity and innate immune responses induced by Nep1-like proteins. Plant Cell 18 3721-3744.

Redman, R.S. et al. (2001). Fungal symbiosis: from mutualism to parasitism, who controls the outcome, host or invader? New Phytologist 151, 705716.

Rooney, H. C. et al. (2005). Cladosporium Avr2 inhibits tomato Rcr3 protease required for Cf-2-dependent disease resistance. Science, 308, 17831786.

Schulze, S. et al. (2016). Effect of sugar beet variety and nonhost plant on Rhizoctonia solani AG2-2IIIB soil inoculum potential measured in soil DNA extracts. Phytopathology 106, 1047-1054. 
Stergiopoulos, I. \& de Wit, P.J.G.M. (2009). Fungal effector proteins. Annual Review of Phytopathology 47, 233-263.

Takahara, H. et al. (2016). Colletotrichum higginsianum extracellular LysM proteins play dual roles in appressorial function and suppression of chitin-triggered plant immunity. New Phytologist 211, 1323-1337.

Utermark, J. \& Karlovsky, P. (2008). Genetic transformation of filamentous fungi by Agrobacterium tumefaciens. Protocol Exchange. DOI: 10.1038/nprot.2008.83.

van den Burg, H.A. et al. (2006). Cladosporium fulvum Avr4 protects fungal cell walls against hydrolysis by plant chitinases accumulating during infection. Molecular Plant-Microbe Interactions 19, 1420-1430.

Vleeshouwers, V.G.A.A. \& Oliver, R.P. (2014). Effectors as tools in disease resistance breeding against biotrophic, hemibiotrophic, and necrotrophic plant pathogens. Molecular Plant-Microbe Interactions 27, 196-206.

Weiberg, A. et al. (2013). Fungal small RNAs suppress plant immunity by hijacking host RNA interference pathways. Science 342, 118-123.

Wibberg, D. et al. (2016a). Draft genome sequence of the sugar beet pathogen Rhizoctonia solani AG2-2IIIB strain BBA69670. Journal of Biotechnology 222, 11-12.

Wibberg, D. et al. (2016b). Genome analysis of the sugar beet pathogen Rhizoctonia solani AG2-2IIIB revealed high numbers in secreted proteins and cell wall degrading enzymes. BMC Genomics 17, 245.

Wibberg, D. et al. (2013). Establishment and interpretation of the genome sequence of the phytopathogenic fungus Rhizoctonia solani AG1-IB isolate 7/3/14. Journal of Biotechnology, 167, 142-155.

Zheng, A. et al (2013). The evolution and pathogenic mechanisms of the rice sheath blight pathogen. Nature Communications, 4, 14. 

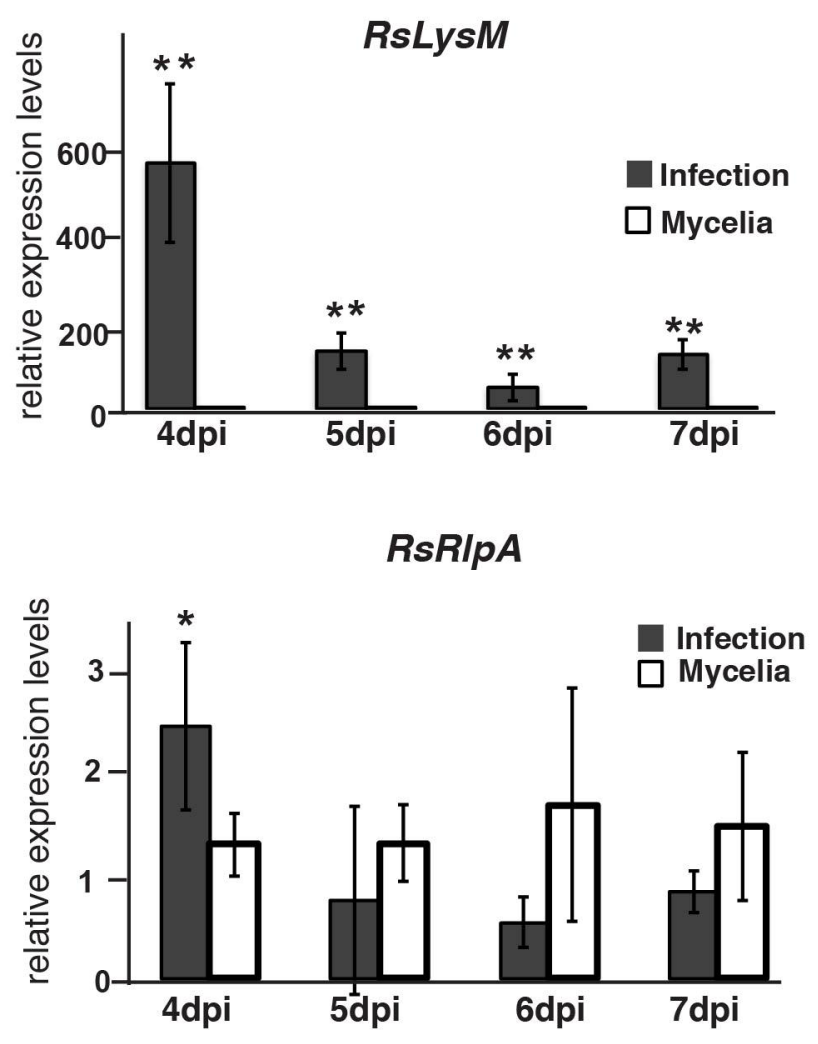

Figure 1. Relative transcript levels of the Rhizoctonia solani AG2-2IIIB genes RsLysM and RsRIpA. Sugar beet plants were grown in infested soil for 4,5,6, and 7 days before harvest for real-time qRT-PCR. Rhizoctonia solani mycelia was grown in PDB medium and used as comparison. The G3PDH gene was used as internal standard. Error bars represent SD based on at least three biological replicates. Asterisks ( ${ }^{*} p$ value $<0.05,{ }^{* *} p$ value $\left.<0.01\right)$ indicate statistically significant differences between columns at the same time point according to Student's T test. 
RsLysM

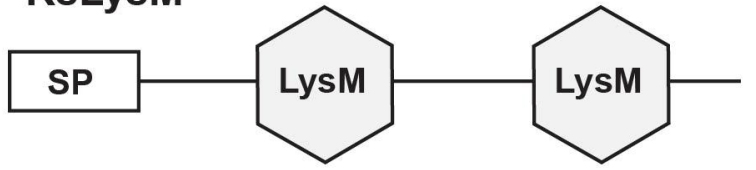

RsRIpA

SP

B

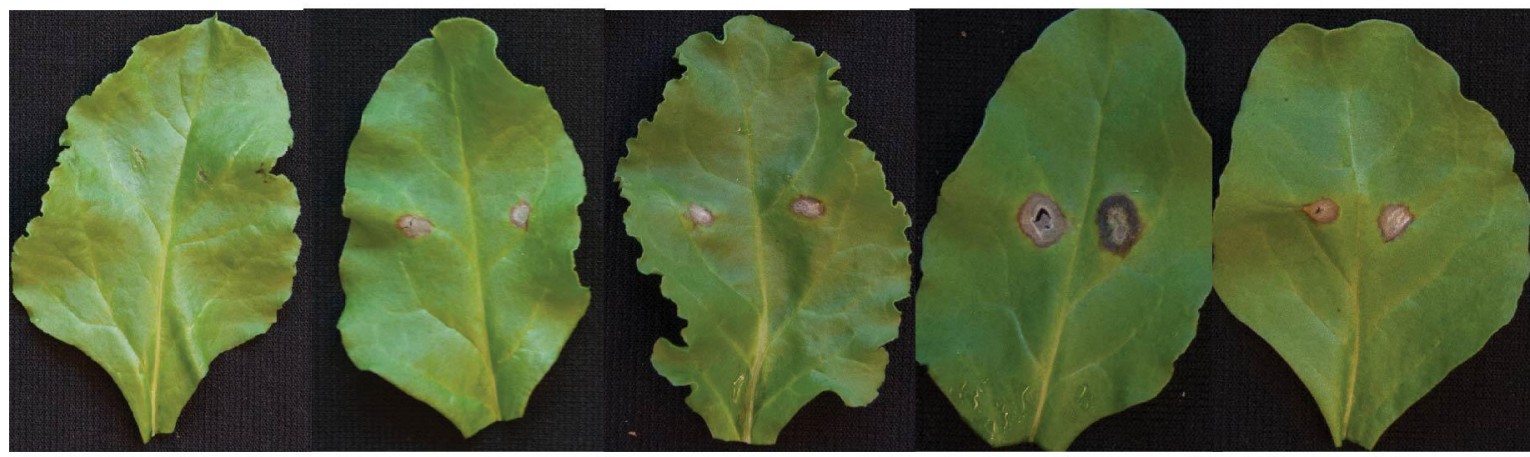

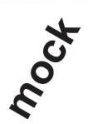

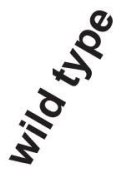

C

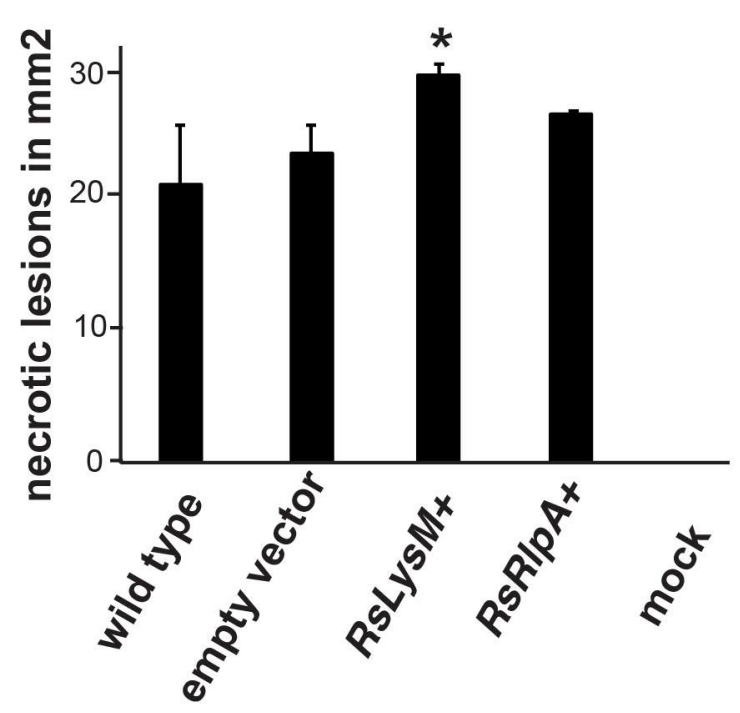

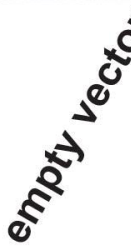
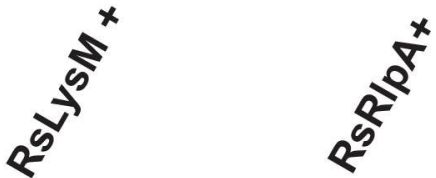

D

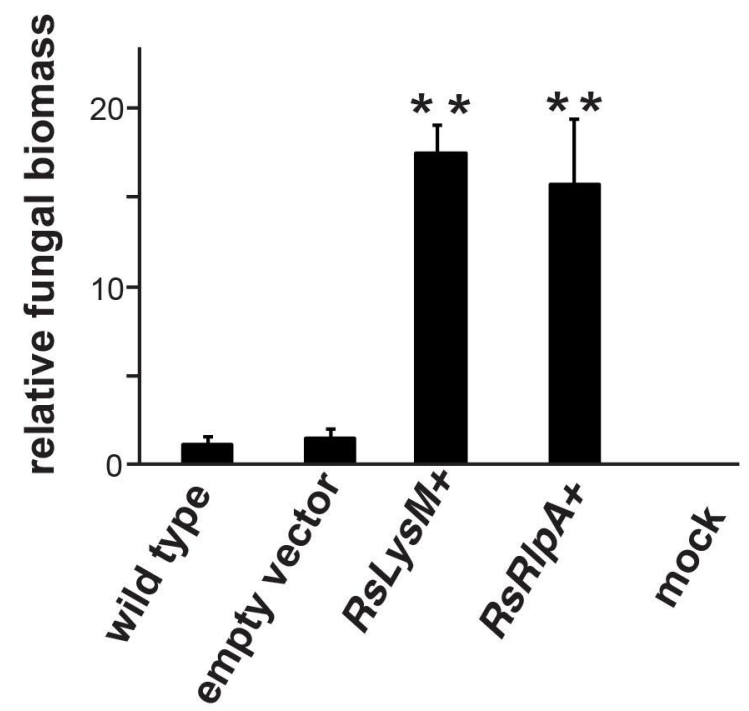

Figure 2. Assays of RsLySM and RsRIpA candidate effectors. (A) Domain structure of RsLySM and RsRlpA proteins. (B) Phenotypes on sugar beet leaves in response to $\mathrm{C}$. beticola strains harboring RsLySM and RsRIpA genes driven by the gdpA promoter, 7dpi. (C) Area of necrotic lesions on sugar beet leaves. (D) Fungal biomass quantification upon infection of sugar beet leaves. For quantitative PCR (qPCR), the C. beticola actin (act) gene was used. Data were normalized with the elongation factor gene (elf-1) from Beta vulgaris. Data show the average of three independent overexpression strains each includes three biological replicates. Asterisks $\left({ }^{*} p<0.05,{ }^{* *} p<0.01\right)$ indicate statistic significant differences between the wild type, empty vector and overexpression $C$. beticola strains according to Fisher's test. Error bars represent SD based on three independent strains. 
RsLySM
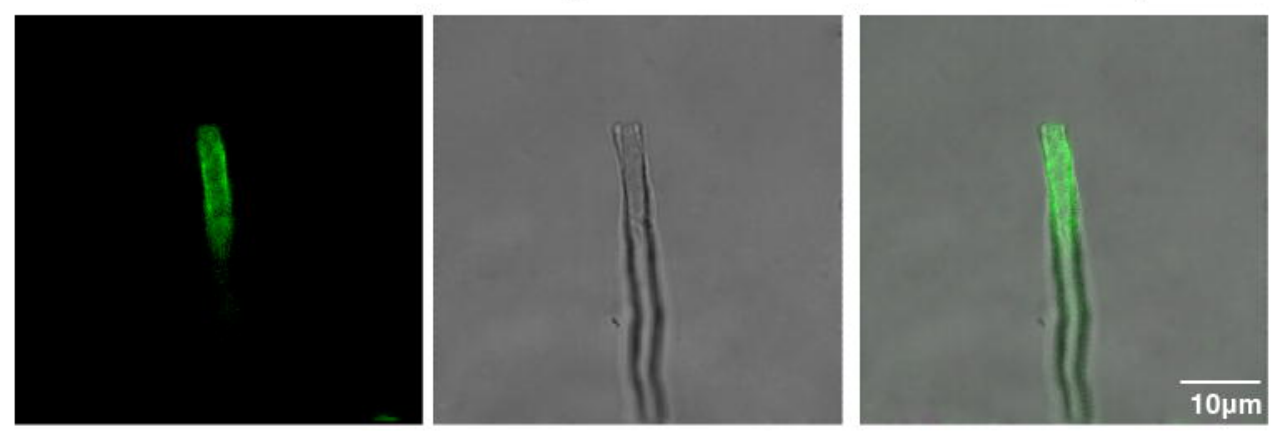

RsRIpA protein
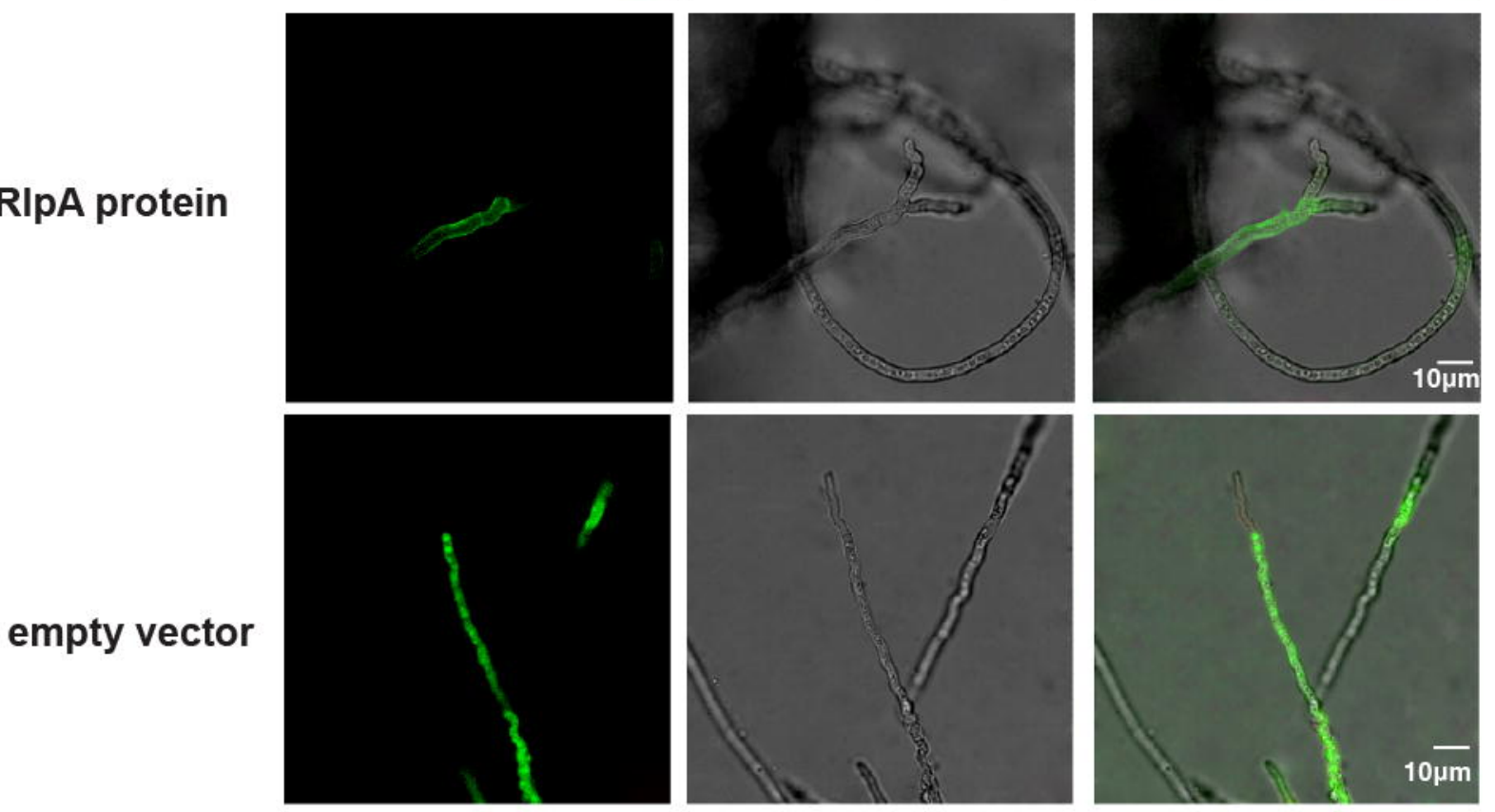

wild type
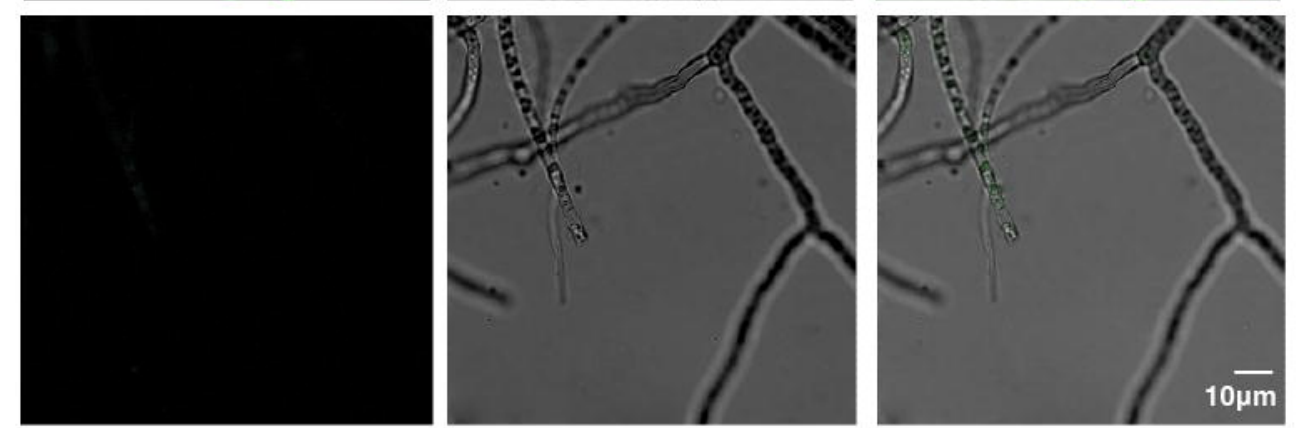

Figure 3. Live-cell imaging of GFP-tagged R. solani RsLySM and RsRIpA effector candidates in $C$. beticola hyphae. Live-cell imaging was performed with a laser-scanning confocal microscope with a sequential scanning mode, 7 days after inoculation on potato dextrose agar. GFP was excited at $488 \mathrm{~nm}$ and collected at $505-525 \mathrm{~nm}$. 
A
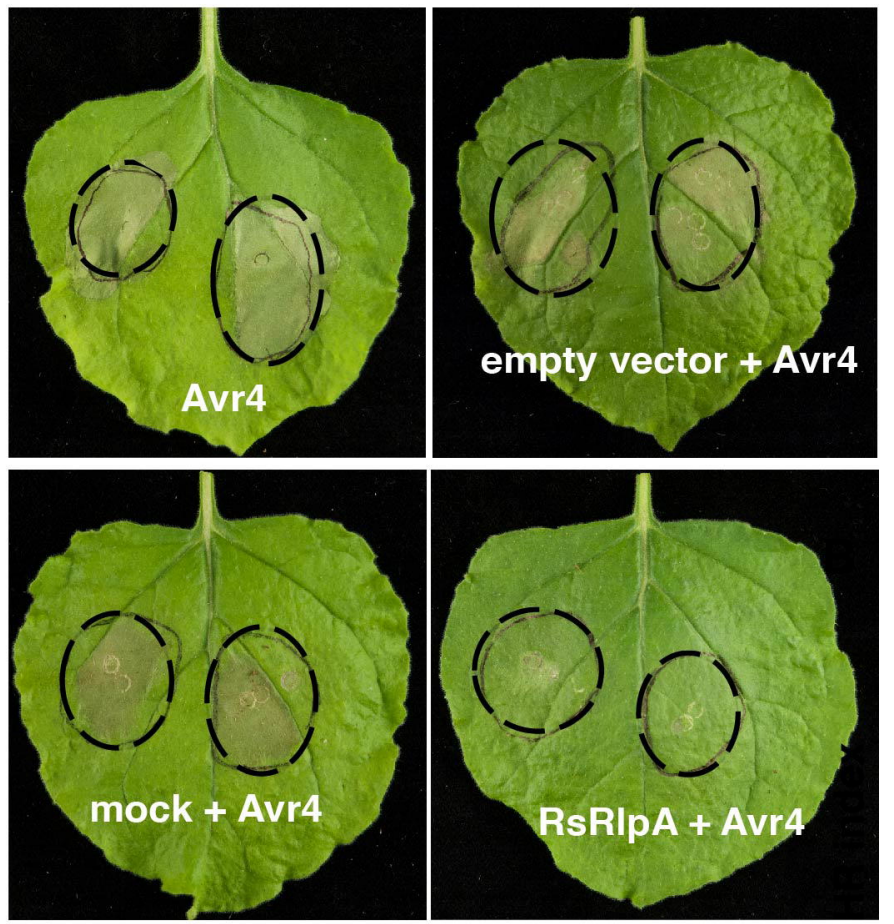

B

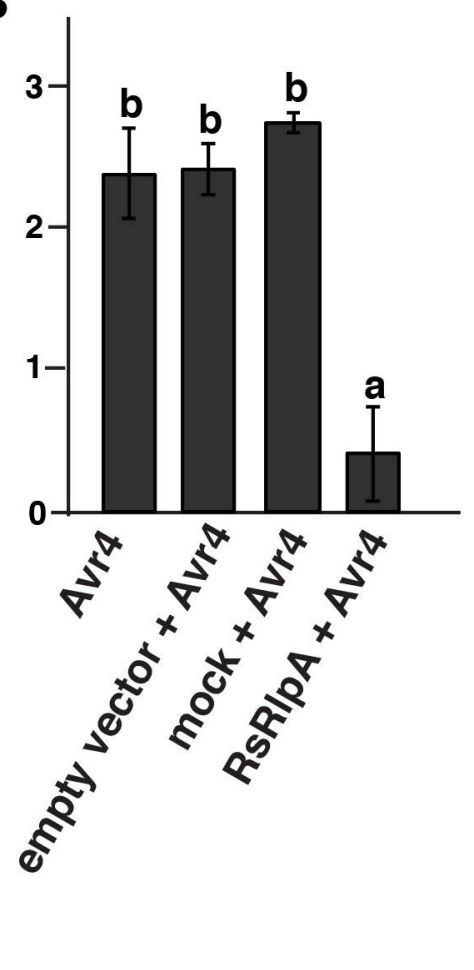

Figure 4. Assay for suppression of Avr4-mediated hypersensitive response (HR). (A) Symptoms on transgenic Nicotiana bethamiana leaves expressing the tomato Cf-4 receptor. (B) HR scale from 0-3 with " 0 " indicates no symptoms and " 3 " severe symptoms on $N$. bethamiana. Leaves were agro-infiltrated first with the RsRIpA effector candidate driven by the CaMV:35S promoter and HR challenged 24hpi with the Avr4 effector. Empty vector and mock inoculation were used as controls. Cycles with dashed lines show the agro-infiltration sites of both effectors. Images taken $3 d p i$. Different letters $(a, b)$ indicate statistical significant differences according to Tukey test $(p<0.05)$. Error bars represent SD of six plants, each contained two agro-infitrated leaves. 

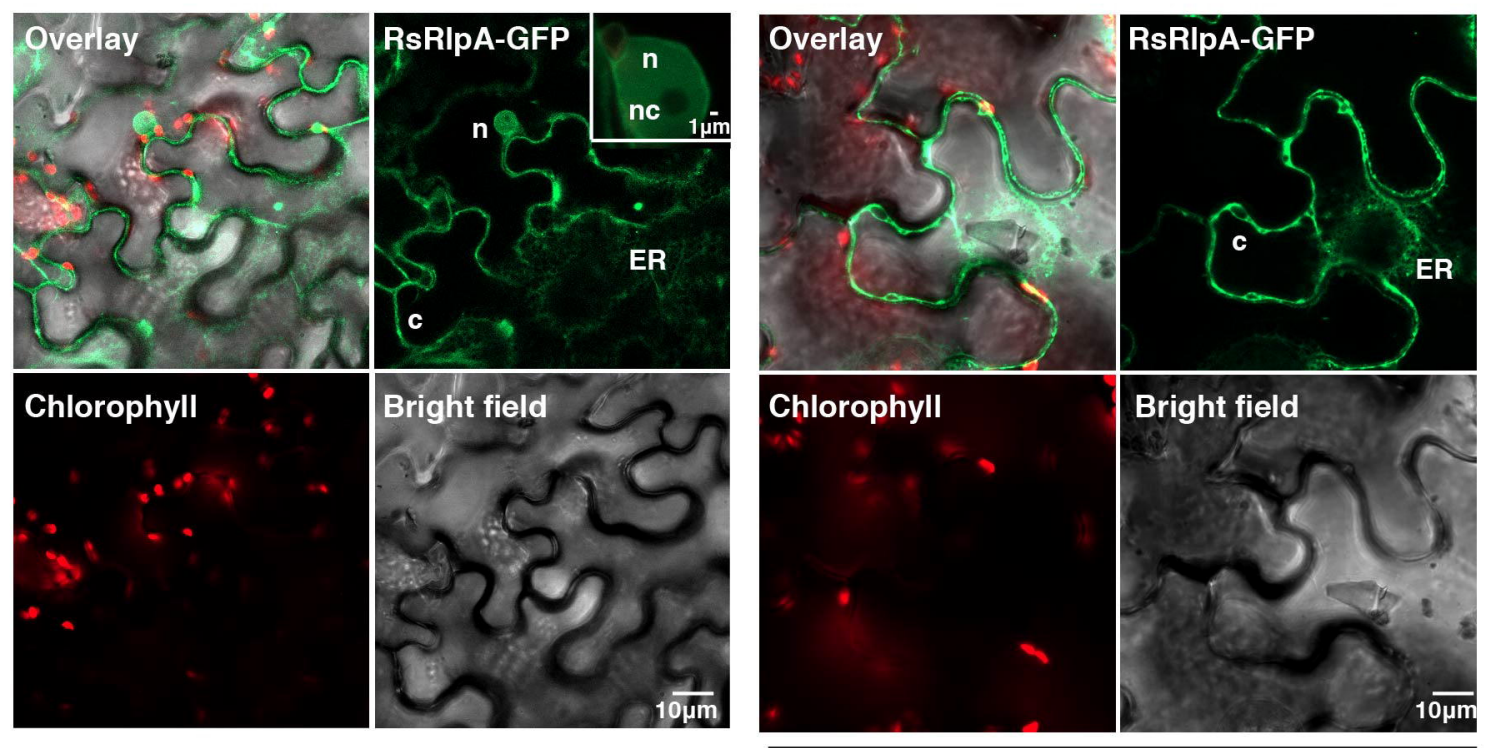

plasmolysis
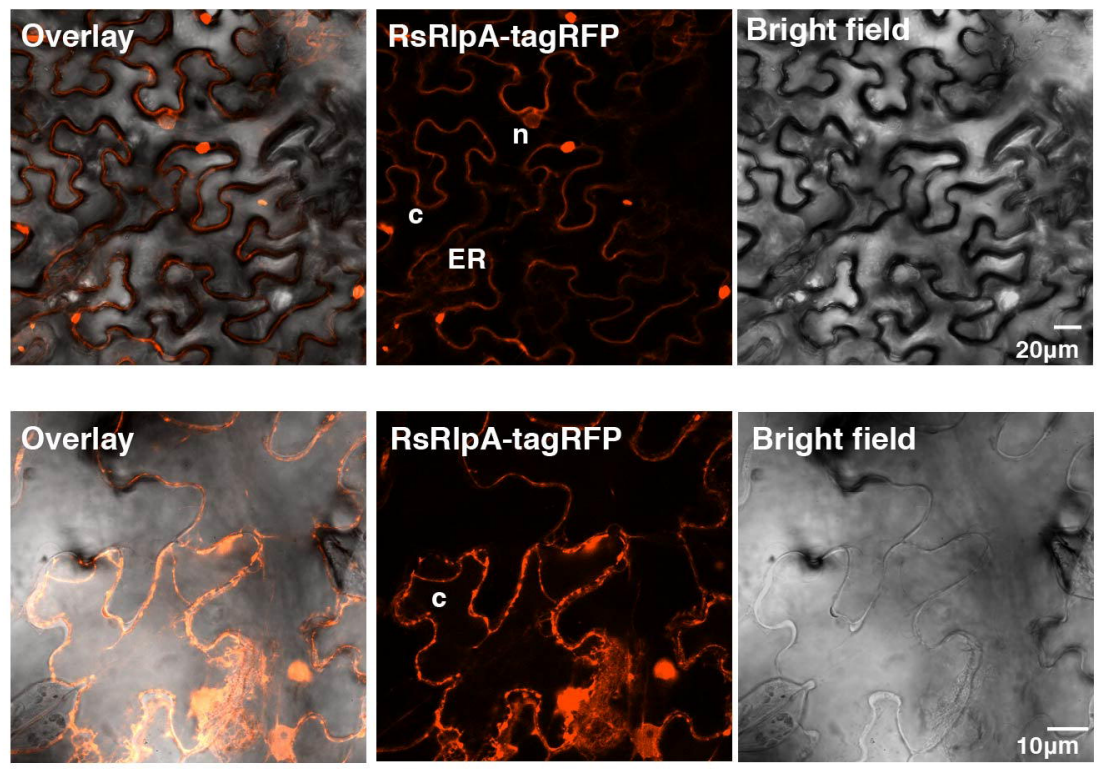

plasmolysis

Figure 5. Live-cell imaging of C-terminal GFP-tagged or tagRFP-tagged $R$. solani RsRIpA effector candidate in agro-infiltrated Nicotiana benthamiana leaves. The localizations were monitored with a laser-scanning confocal microscope with a sequential scanning mode $48 \mathrm{hrs}$ post infiltration. The GFP and the chlorophyll were excited at $488 \mathrm{~nm}$. GFP (green) and chlorophyll (red) fluorescent signals were collected at $505-525$ and $680-700 \mathrm{~nm}$, respectively. The tagRFP was excited at $558 \mathrm{~nm}$ and collected at $545-620 \mathrm{~nm}$. Plasmolysis was incited by using $1 \mathrm{M}$ mannitol for 30 min. (c): cytoplasm, (ER): endoplasmic reticulum, (n): nucleoplasm, (nu): nucleolus. 
A

bioRxiv preprint doi: htts

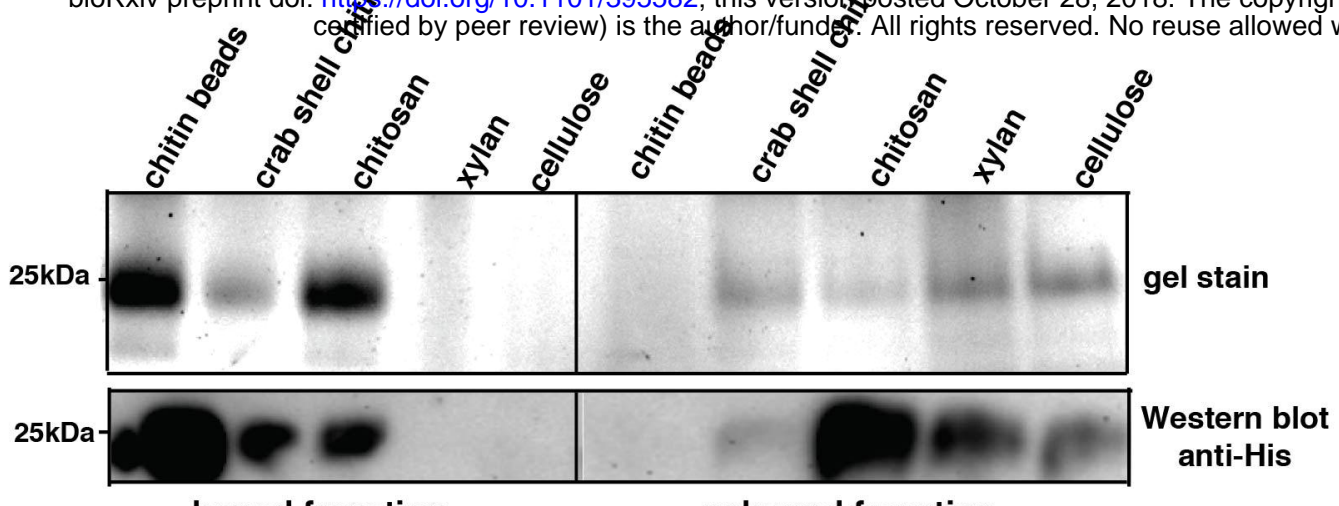

bound franction

unbound franction

B

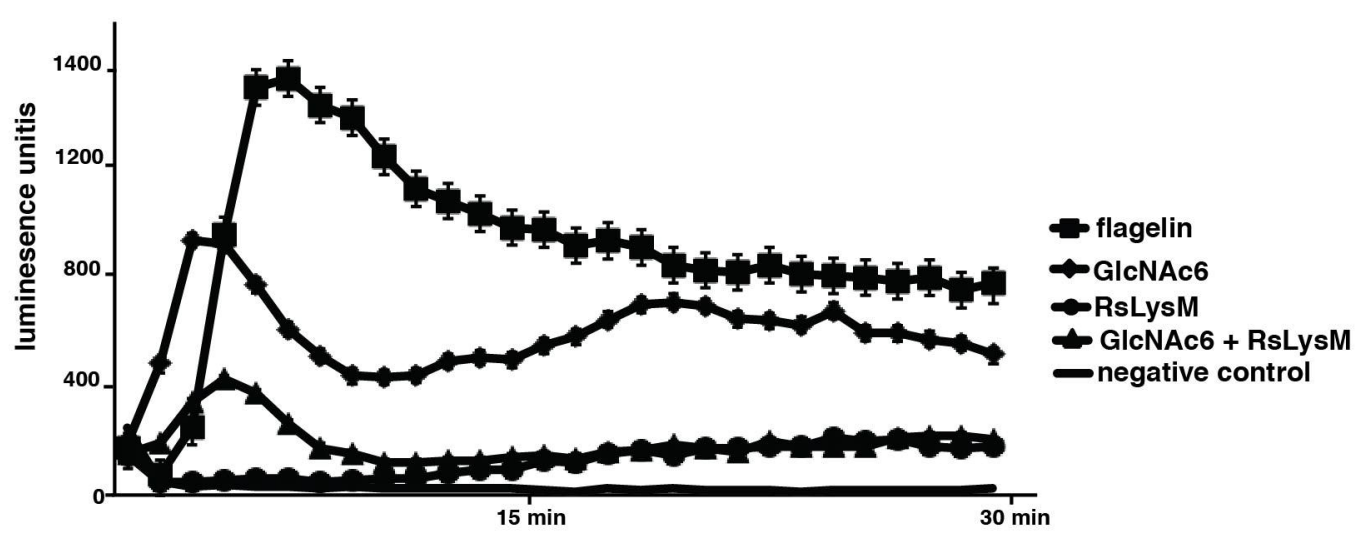

C

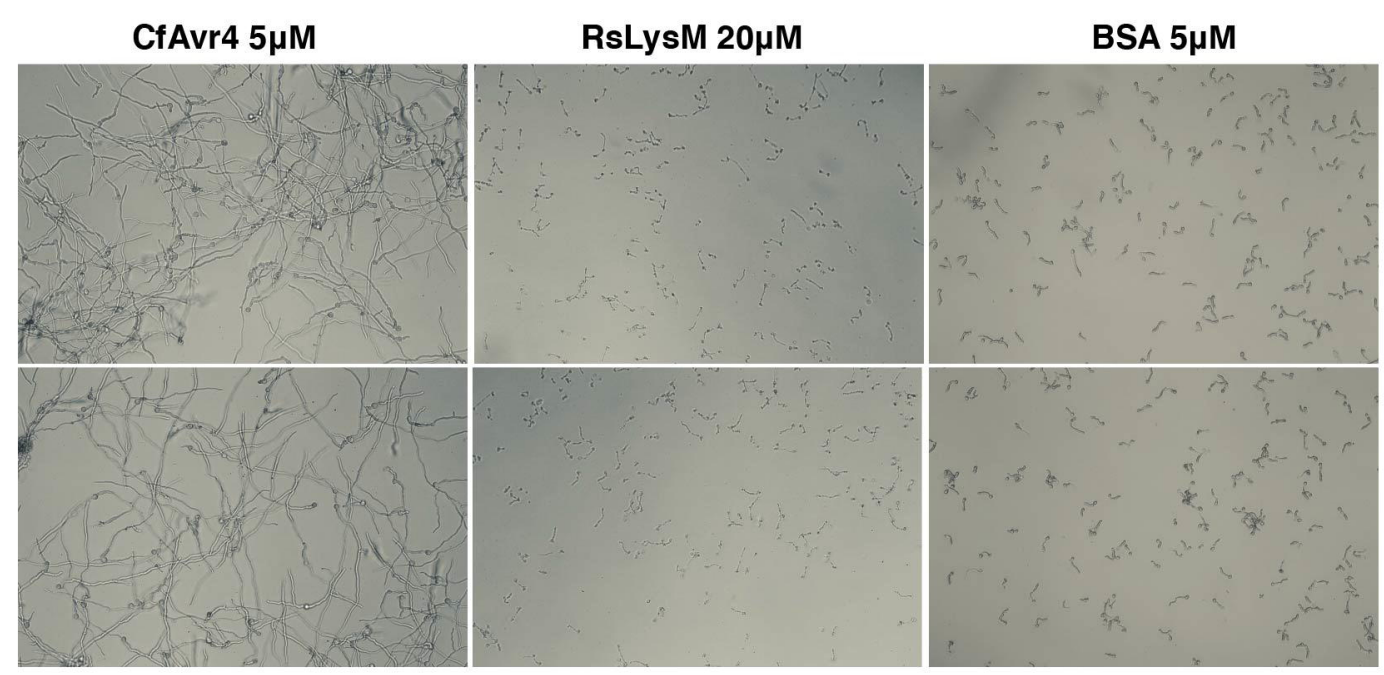

Figure 6. Functional analysis of the RsLysM protein. (A) Binding affinity assay on different insoluble polysaccharides. $20 \mu \mathrm{g} / \mathrm{ml}$ of LysM protein was mixed with $5 \mathrm{mg}$ of chitin beads, crab shell chitin, chitosan, xylan or cellulose. Presence of protein in bound (pellet) and unbound fraction (supernatant) separated using SDS-polyacrylamide gel electrophoresis followed by Coomassie staining. (B) Chitin-induced oxidative (ROS) burst assay in leafs. Production of ROS was determined using luminol-dependent chemiluminescence. Leaf discs were treated with

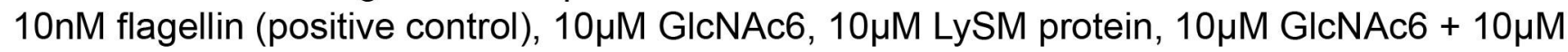
LySM protein. The luminol reagent was used as a negative control. At least eight biological replicates were run, and experiment repeated twice. (C) Hyphal protection assay. $20 \mu \mathrm{M}$ of LysM protein was incubated with $3 \times 10^{5} \mathrm{~T}$. virens conidia for $30 \mathrm{~min}$ and $0.1 \mathrm{U}$ bacterial chitinase and $10 \mathrm{U}$ zymolyase. Images were taken 6hpi. $5 \mu \mathrm{M}$ Avr4 or BSA were used as positive and negative controls respectively. Assay was repeated twice with same results. 